\title{
苔藓植物岩溶作用研究进展
}

\author{
蒙文萍 1 , 戴全厚 $^{1 *}$ 再景丞 $^{3}$
}

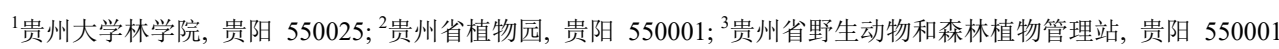

摘 要 苔藓植物参与岩溶作用的过程是生物岩溶的重要内容。开展苔藓植物岩溶作用研究可为石漠化裸岩的生态恢复及其 综合治理提供理论依据和技术支撑。该文系统论述了苔藓植物岩溶作用的过程(溶蚀与沉积)、机理及其与生境互作机制的最 新研究进展。苔蘚植物及其生物结皮在干湿交替下发生膨胀、卷缩、冻融等物理机械力, 以及其代谢分泌物和因呼吸作用而 产生的 $\mathrm{H}_{2} \mathrm{CO}_{3}$ 与岩石中矿物反应, 破坏矿物晶体结构, 导致矿物裂解、使岩面崩解脱落而改变岩面形态, 塑造岩溶地貌, 形成 原始土壤。苔藓植物岩溶作用驱动力的大小与植物功能性状、基岩性质与生存环境密切相关。此外, 生物岩溶作用需要长时 间尺度的监测, 研究周期长, 建议建立野外长期监测基地, 加强对其内在机制与过程以及与生境间互作机制, 苔藓植物的生 理代谢过程及其生物酶等与岩溶作用的相互关系, 岩溶地区苔藓植物环境适应性特征及其生物多样性维持机制等方面的研究。

关键词＼cjkstart苔蘚植物; 岩溶作用; 过程; 机理; 生境; 互作机制

蒙文萍, 戴全厚, 苒景丞 (2019). 苔藓植物岩溶作用研究进展. 植物生态学报, 43, 396-407. DOI: 10.17521/cjpe.2019.0020

\section{A review on the process of bryophyte karstification}

MENG Wen-Ping ${ }^{1,2}$, DAI Quan-Hou ${ }^{{ }^{*}}$, and RAN Jing-Cheng ${ }^{3}$

${ }^{1}$ College of Forestry, Guizhou University, Guiyang 550025, China; ${ }^{2}$ Guizhou Botanical Garden, Guiyang 550001, China; and ${ }^{3}$ Guizhou Wildlife and Forest Plant Management Station, Guiyang 550001, China

\begin{abstract}
Bryophyte participate in karstification is an important part of biokarst process. Numerous studies on bryophyte karstification provide theoretical and technical foundation for restoration and comprehensive management of bare rock in the rocky desertification area. This article systematically reviewed the process (dissolution and sedimentation), mechanism and interaction relationship between bryophyte karstification and habitats. Bryophyte and its biological crusts emerge physical forces such as expansion, curling, freezing and thawing when they are under alternating wet or dry conditions can destroy rock. In addition, their metabolic secretions and $\mathrm{H}_{2} \mathrm{CO}_{3}$ formed by respiration, which react with minerals resulting in destruction of the crystal structure, pyrolysis the minerals, further the rock surface disintegrated and the surface morphology changed, the karst landform and the original soil formed. The driving force of bryophyte karstification closely related to plant functional traits, rock properties and habitat. Studies on biokarst need long-term monitoring and long research period. It is recommended to establish a long-term monitoring sites for strengthening examinations on process, internal mechanisms, and interaction relationship with habitat of bryophyte karstification. At the same time, physiological metabolic processes of bryophytes and the relationship with bryophyte karstification should be emphasized. The environmental adaptability of bryophyte and the maintenance mechanism of biodiversity in karst areas need research attention as well.
\end{abstract}

Key words bryophytes; karstification; process; mechanism; habitat; interaction mechanism

Meng WP, Dai QH, Ran JC (2019). A review on the process of bryophyte karstification. Chinese Journal of Plant Ecology, 43, 396-407. DOI: $10.17521 /$ cjpe.2019.0020

生物岩溶是指由生物引起的岩溶作用现象及 其产物，即生物溶蚀、破坏可溶岩和沉淀形成次生 化学沉积物及其所遗留或形成的形态(Schneider,
1976)。生物岩溶作用(溶蚀作用和沉积作用)激活了 岩溶生态系统基质中的物质和能量，使各个圈层间 信息交流更加频繁与复杂。苔藓植物作为生态系统

收稿日期Received: 2019-01-22 接受日期Accepted: 2019-05-06

基金项目: 国家自然科学基金(41671275)、国家重点研发计划(2016YFC0502604)和国家自然科学基金委员会-贵州人民政府喀斯特科学研究中心项 目(U1812401)。Supported by the National Natural Science Foundation of China (41671275), the National Key R\&D Program of China (2016YFC0502604), and the National Nature Science Foundation of China and the Karst Science Research Center of Guizhou Province (U1812401).

* 通信作者Corresponding author(qhdairiver@163.com) 
演替过程中的先锋植物(薛建辉, 2013), 通过生物岩 溶作用改善裸岩生境, 为草本和灌木的出现奠定了 物质基础(Harper \& Belnap, 2001; Jackson, 2015), 对 岩溶生态系统的维持与发展具有重要意义( Li et al., 2006; Costa et al., 2018)。苔蘚植物岩溶作用已成为 岩溶生态学研究的热点。因此, 了解和掌握岩面苔 藓植物功能性状特征、岩溶作用机理及其与环境间 的互作机制对研究岩溶地区的生物多样性、石漠化 生态恢复、岩溶碳汇以及丰富生物岩溶理论等意义 重大。然而, 苔藓植物岩溶作用研究因涉及学科众 多、问题复杂, 还未进入系统化研究阶段。为了破 除各学科间的壁垒, 促进苔藓植物岩溶作用研究更 进一步发展, 本文根据近年来有关苔藓植物岩溶作 用的文献资料, 从以下方面综述苔藓植物岩溶作用 的研究进展。

\section{1 岩面苔藓植物功能性状特征}

苔藓植物常以丛集型、交织型、匍匐型等着生 于岩面(图1), 假根根系不发达, 只起固着作用(娄玉 霞, 2013), 其特殊的叶表面结构和细胞特征(娄红祥, 2012), 使苔藓具有耐高温、抗旱、蓄水能力强、保 湿固土等功能(Proctor \& Pence, 2002; Krol et al., 2003; Zhang et al., 2017)。曹建华和袁道先(1999)研 究发现, 当岩石表面有苔藓植物时, 其蒸发失水和 主动吸水时间比裸岩分别延长了 $48 \%$ 和 $57 \%$, 持水 量提高了16.6倍, 分析认为苔藓植物可以延缓岩面 水分蒸发时间, 增加岩石水化学作用时间, 但是苔
藓植物的这种蓄水保湿能力存在明显的种间差异 (申家琛等, 2017; 张显强等, 2018a)。苔藓植物参与 岩溶作用的另一大优势，是其叶表面角质层不发育， 且阳离子交换位点多(吴玉环等, 2001), 能够吸附多 种阳离子, 这些阳离子在苔藓植物体内被转化为无 机盐、有机盐以及其他形式的化合物储存于组织中。 苔藓植物除了以生物化学方式对岩石进行溶蚀之外, 还可以通过物理机械作用破坏岩石。例如，苔藓植 物的假根可以伸入岩石裂隙，对岩石有一定的破坏 作用(Jackson, 2015)。再者, 当气候干旱时, 苔藓植 物在卷缩过程中会拔起岩面的一些小颗粒, 从而加 速了岩石被破坏和成土速度(连宾等, 2008)。此外, 苔藓植物常与藻类、地衣、微生物等共生形成生物 结皮, 它们之间的相互关系以及因气候因素引起的 干湿交替和冻融作用对岩石也具有一定的破坏性 (郑云普等, 2009)。例如, 菌类的菌丝可以伸入岩石 晶体结构中破坏岩石, 细菌与真菌形成的配合基能 够络合三价铁离子, 细菌分泌的柠檬酸、乙酸以及 地衣分泌的地衣酸(连宾等, 2011), 可以溶解岩石中 的矿物(Won-Pyo et al., 2016; Wang et al., 2017)。因 此在研究苔藓植物岩溶作用时, 应明确苔藓植物自 身及其与其他生物形成的生物结皮两者之间的关 系。目前, 有关岩面苔藓植物功能性状的研究主要 集中在小范围内特定生境中的苔藓植物研究, 无法 代表岩溶生态系统苔藓植物的整体情况。对于大尺 度空间上，因生境异质性高而造成相同物种在形态 结构、光合特征及其钲 $(\mathrm{Ca})$ 、碳 $(\mathrm{C})$ 、氮 $(\mathrm{N})$ 、磷 $(\mathrm{P})$
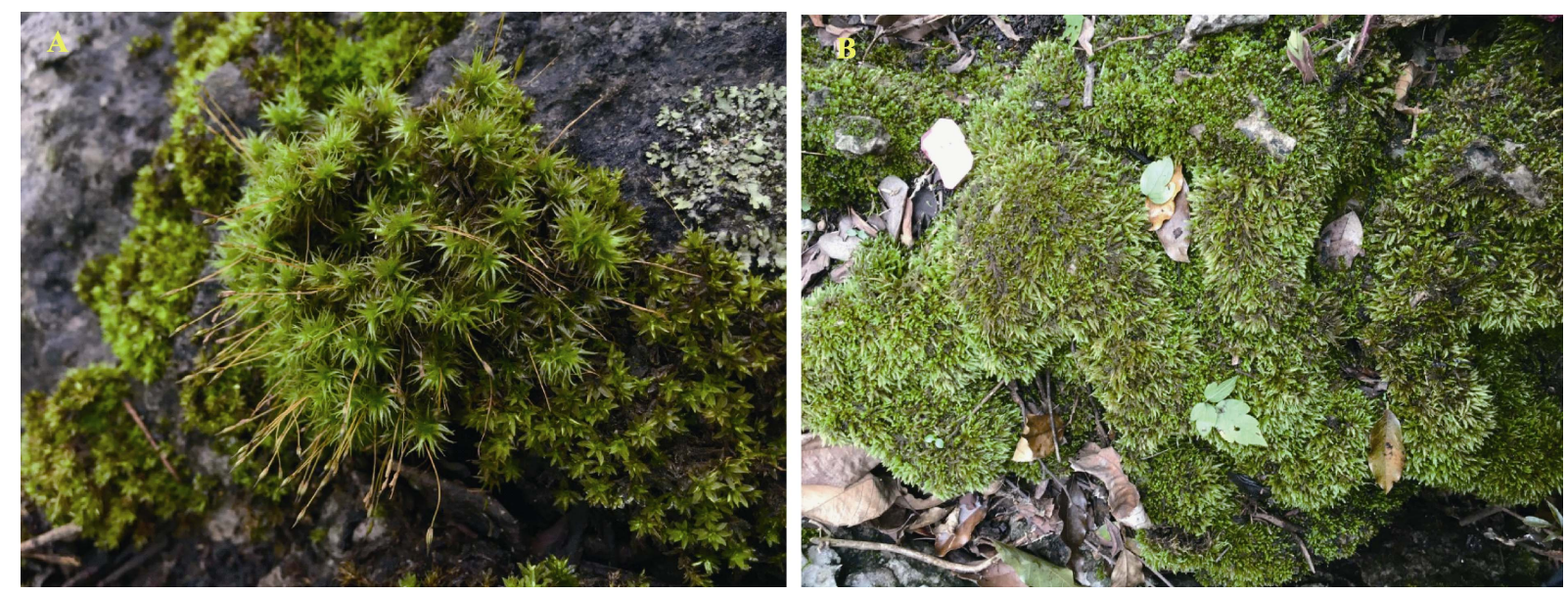

图1 贵州普定喀斯特生态监测站岩面苔藓植物。A 中的苔藓植物主要是多枝缩叶藓、卷叶湿地藓。 $\mathbf{B}$ 中的苔藓植物主要是平 蒴蘚、皱叶牛舌藓、狭网真藓。

Fig. 1 Bryophytes on rock surface at Puding karst ecological monitoring station in Guizhou. A, mosses of Ptychomitriaceae gardenri and Hyophila acutifolia. B, mosses of Plagiobryum zierii, Bryum anagustirete and Anomodon rugelii. 
等元素的代谢调节机制等方面的差异性的研究还未 引起人们的广泛关注。岩溶生态系统的物质基础相 对贫乏, 具有“缺氮少磷钙丰富”的特征。因此, 研究 Ca、C、N、P等元素在苔藓植物体内的代谢机制对 揭示岩溶生态系统苔藓植物和其他植物的生物多样 性维持机制, 及其岩溶作用特征具有重要的科学 意义。

\section{2 苔藓植物对岩石的溶蚀作用}

\section{1 苔蘚植物溶蚀作用原理与过程}

苔藓植物对岩石的溶蚀作用原理与过程实质是 岩石中矿物与苔藓植物之间的作用过程(图2)。苔藓 植物因呼吸作用形成的 $\mathrm{H}_{2} \mathrm{CO}_{3}$ 和代谢分泌物与岩石 中的矿物发生酸化、碱化、氧化还原、螯合等反应, 随着反应过程不断推进, 岩石矿物成分发生变化, $\mathrm{Ca}$ 、镁 $(\mathrm{Mg})$ 、钠 $(\mathrm{Na}) 、$ 钾 $(\mathrm{K}) 、$ 铁 $(\mathrm{Fe}) 、$ 铝 $(\mathrm{Al})$ 、硅 $(\mathrm{Si})$ 等从碳酸盐、硅酸盐、铝酸盐、硫化物、氧化物 中溶出, 破坏原岩晶体结构, 达到溶蚀岩石, 促使
风化的目的(Street et al., 2013; Li et al., 2015; Lammers et al., 2017)。这种岩溶驱动力的大小因植物形 态特征、岩石性质与生存环境改变而发生变化。有 研究表明, 碳酸盐岩溶蚀过程中, 生物成因的 $\mathrm{CO}_{2}$ 占溶蚀强度的 $49.26 \%$, 有机酸占 $37.11 \%$ (张楷燕, 2017)。苔藓植物溶蚀作用过程释放的矿物离子和其 他元素部分被苔藓植物拦截形成岩溶沉积物; 部分 被苔藓植物吸收利用, 转化为其他物质存储于植物 体内; 其余则随降雨冲刷而流失, 进入其他生态系 统。关于苔藓植物呼吸作用和其分泌物引起的岩溶 作用驱动力、效率及其与苔藓植物生活型、种间差 异、生境特征之间的相互关系仍未引起人们的关注， 对苔蘚植物溶蚀作用过程和机理、苔藓植物对岩石 中矿物营养元素释放规律的影响, 以及这些矿物质 在岩溶沉积过程中的形态转化过程及其与生境间的 相互作用关系等的研究还未见报道, 这也正是揭示 苔藓植物岩溶作用机理的关键点。岩石的溶解速率 与有机酸的酸性和溶蚀能力密切相关(刘胜亮等,

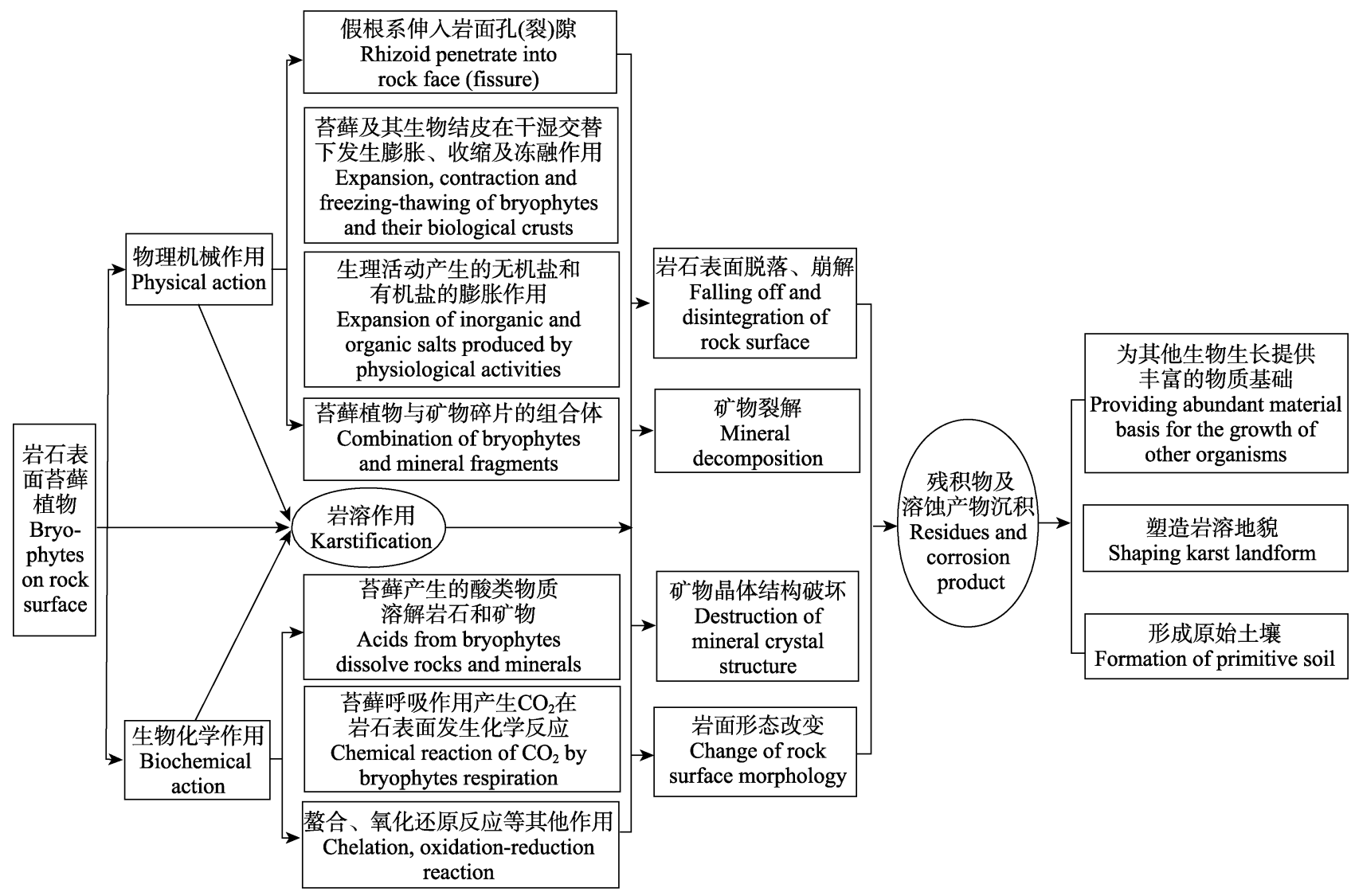

图2 苔藓植物岩溶作用过程示意图。岩石表面的苔藓植物通过物理机械力与生物化学作用力破坏和溶蚀岩石, 从而改变了 岩面形态, 塑造了岩溶微地貌, 溶蚀产物的沉积形成了原始土壤。

Fig. 2 A schematic flowchart showing the bryophyte karstification process. The moss on rock surface through physical and biochemical action to destroy and corrosion the rock, change the rock surface morphology and form the karst microtopography. At the same time, the dissolution products are deposited to form the original soil.

www.plant-ecology.com 
2017), 然而有机酸的理化特性取决于其官能团的 属性(赵志曼等, 2018)。因此, 今后需结合有机化学、 气相色谱质谱(GC-MS)、核磁共振(NMR)、液相色 谱(HPLC)等技术手段深入研究苔蘚植物的溶蚀过 程与机理。

此外, 苔藓植物体内的碳酸酐酶(CA)对岩溶作 用也有促进作用(Smith \& Griffiths, 2000), 它主要催 化 $\mathrm{CO}_{2}$ 的水合反应: $\mathrm{CO}_{2}+\mathrm{H}_{2} \mathrm{O} \rightleftharpoons \mathrm{HCO}_{3}^{-}+\mathrm{H}^{+}$(吴雁 雯和张金池, 2015)。李为等(2005)研究发现无 CA催 化时上述可逆反应达到平衡的时间是有 $\mathrm{CA}$ 催化时 的 $6 \times 10^{7}$ 倍。生物体内均含有 CA, 且CA活性因物种 的不同而存在显著差异, 细菌、真菌、放线菌内均 含有 $\mathrm{CA}$, 且微生物细胞内CA活性不同于细胞外 CA, 细菌的CA对碳酸盐岩系统碳储存贡献在 $20 \%$ 以上 (Li et al., 2006), 真菌的CA对白云岩的溶蚀能力强 于对石灰岩(申泰铭等, 2014; 申泰铭, 2018)。那么苔 藓植物体内 CA 对岩溶作用的贡献是多少? 各组织 内的CA活性是否也存在这种差异? 对此以及关于 苔藓植物体内 CA 代谢的调节机制及原理仍不得而 知。目前, 关于苔藓植物 $\mathrm{CA}$ 的研究, 主要集中在各 类苔藓植物CA含量、活性及其对岩石溶蚀过程的促 进作用等方面。例如, 刘天雷等(2017)研究发现, 虽 然苔藓植物都会分泌CA, 但CA分泌量、速率和活 性存在种间差异。这种差异性可能是因生活型或者 生长环境的不同而导致苔藓植物生理代谢与功能性 状的差异引起的。

\section{2 苔藓植物溶蚀产物分析}

岩石经苔藓植物溶蚀后, 表面常常出现一些凹 凸不平和微溶孔, 这是由于岩石成分不均匀, 导致 生物钻孔力的差异性(王福星等, 1998), 以及溶蚀产 物流失而形成溶蚀微地貌。王福星等(1993)将这些 溶蚀微地貌, 按照口面形态、直径大小、深度等, 把 短径小于 $1 \mathrm{~mm}$ 的命名为微溶孔, 把短径为 $1-20 \mathrm{~mm}$ 的命名为溶孔, 把短径为 $20-100 \mathrm{~mm}$ 的命名为溶 坑。付兰和张朝晖(2010)将连续分布的苔藓植物溶 蚀微地貌, 按测算结果, 把长7.7-14.4 $\mathrm{cm}$ 的命名为 溶丝; 长 $0.8-19.0 \mathrm{~cm}$, 宽0.6-7.2 $\mathrm{cm}$ 的命名为溶斑; 长6.0-24.5 cm, 宽1.2-2.2 cm 的命名为溶带。苔藓植 物溶蚀过程很难通过宏观手段观测, 导致这两种命 名法在大小和形态方面存在明显差异。然而, Haward等(2011)借助原子力显微镜(AFM)观察到黑 云母表面浅层溶蚀坑的形成过程, 通过分析图像计
算质量损失, 从而确定溶蚀过程中黑云母表面的活 化能大小。研究表明, 通过分析活化能得到的溶坑 大小与测量其周长所得的数据一致。因此, 在研究 溶蚀微地貌时, 应采用高精度仪器, 统一测量标准, 分析微地貌是苔藓植物溶蚀作用产生, 还是由于淋 溶风化而形成, 有利于后期岩溶微地貌之间的对比 分析和进一步深入研究。这些微地貌的形态特征与 生物类型和发育生境密切相关(蔡胡霖, 2017), 在温 湿度优越的地区发育更为广泛(Liang \& Xu, 2014; Liu et al., 2016)。例如, 溶孔、溶雉多发生在低湿度、 强光照、高温环境中, 而溶沟多是在高温、强光和 pH较高的生境内发育(申家琛等, 2018a)。曹建华和 王福星(1996)认为苔藓植物岩溶微地貌与岩溶地区 的宏观地貌形态之间存在一定的相关性, 然而目前 这种关联性以及苔藓植物微地貌之间的关系和发育 方向尚未得到科学揭示。

在岩石溶蚀过程中, 有些岩石因产状、矿物成 分、气候、生物等因素利于溶蚀产物残留, 沉积物 较多, 进而为沉积作用做贡献; 然而有些岩石可溶 性矿物含量多, 则溶蚀残留物较少。例如, $\mathrm{H}_{2} \mathrm{CO}_{3}$ 与 碳酸盐岩中的 $\mathrm{CaCO}_{3}$ 反应生成 $\mathrm{Ca}\left(\mathrm{HCO}_{3}\right)_{2}$ 随水溶失, 残留物较少。有机酸常常与岩石中钙等矿物结合生 成有机酸钲等难溶化合物(张大庚等, 2015), 赋存于 残积物中, 进而影响土壤的矿物组分与含量。由于 生物岩溶作用研究需要长时间尺度的监测, 研究难 度较大, 所以对岩面因苔藓植物溶蚀作用而形成的 溶蚀残积物中的物质种类、形态特征以及苔藓植物 岩溶作用与生境之间关系的研究仍然很欠缺。

\section{3 苔藓植物对岩溶沉积贡献}

岩溶地区土壤的形成和发育是碳酸岩长期溶 蚀、风化与生物富集过程的结果, 其一方面受岩石 结构、性质影响, 另一方面也与植被作用密切相关 (张美良和邓自强, 1994)。研究表明, 碳酸盐岩的成 土速率与岩石溶蚀速率成正比(曹建华等, 2004), 与 酸不溶物含量密切相关(曹沁智等, 2018)。苔藓植物 殖居于岩面, 假根系以及植物体的干湿交替冻融现 象所产生的物理机械力作用于岩石, 产生岩石碎 屑。同时, 有机物与矿物离子络合常常形成难溶物, 这些难溶物与苔藓及其生物结皮死亡残体黏结在一 起, 不仅增加了土壤沉积量, 而且促进有机质积累, 增加土壤养分(朱显谟, 1995; 刘天雷等, 2017)。苔藓植 
物通过影响下垫面土壤湿度, 促进土壤对岩石的岩溶 作用，间接地影响沉积过程。同时，苔藓植物的吸附 拦截功能对防治岩石表面水土流失具有重要作用(李 军峰等, 2013; 贾少华等, 2014; 张显强等, 2018b)。 苔蘚植物的溶蚀作用为岩面土壤形成提供了原始物 质, 关于苔藓对土壤沉积的贡献率与苔藓植物作用 下岩面土壤的形成过程, 以及在此过程中岩石的物 质、能量转化和土壤养分的变化等还未见报道。

苔藓植物沉积作用的另一表现是石灰华或钲华 (张捷, 1993; Kleinteich et al., 2017)。钙华形成的物 理化学过程实质是 $\mathrm{CaCO}_{3}$ 的沉淀与 $\mathrm{CO}_{2}$ 的脱气。苔 藓植物在钙华形成过程中为 $\mathrm{CaCO}_{3}$ 晶体提供了稳定 的生长发育基础, 并且苔藓植物的形态结构控制着 沉积物的形态变化(Miliša et al., 2006), 且它们的发 育形态随沉积环境而变化(张朝晖等, 2004; 郭云等, 2018)。如在洞穴弱光带一般形成洞穴石刺、向光钟 乳石、洞口叠层石等, 这类形态有很强的方向性(王 福星等, 1998)。苔藓植物在水生环境中也常常形成 多样的沉积形态, 如凤尾藓(Fissidens)钙华、石灰藓 (Hydrogonium) 钙华形成的溪流瀑布沉积, 泽藓 (Philonotis)钙华、水灰蘚(Hygrohypnum)钙华形成的 河流瀑布沉积, 地钱-真藓(Marchantia-Bryum) 钙华 等形成的石灰华坝沉积, 以及净口藓(Gymnostomum) 钲华等形成的瀑布洞穴沉积(张朝晖和陈家 宽, 2007)。同时, 也有研究发现, 水流环境也是影响 苔藓钙华沉积的重要因素, 苔藓与钲华层之间的水 流速度与钙华沉积速率呈正相关关系(Wang et al., 2014)。钙华作为大陆古气候的潜在档案, Yan等 (2017)利用碳氧稳定同位素比率 $\left(\delta^{13} \mathrm{C}\right.$ 和 $\left.\delta^{18} \mathrm{O}\right)$, 研究 钙华在沉积过程中碳、氧的循环规律, 分析认为, 钲 华沉积与季节更替引起的气温、降水等的变化密切 相关。目前对于苔藓植物沉积作用的研究主要集中 在沉积形态以及成土量方面, 对于苔藓植物沉积速 率, 对岩溶地区成土过程中的贡献, 以及岩溶沉积 作用对生境的改造和环境对岩溶沉积作用的影响等 方面的研究还未见报道。

\section{4 影响苔藓植物岩溶作用的因素及其作用 机理}

\section{1 湿度、温度、光照及其综合因子}

苔藓植物群落分布特征与生境条件密切相关 (Heino \& Virtanen, 2006; 刘艳等, 2015; Mod et al.,
2016; 李泽科等, 2018; 庞嘉鹏等, 2018), 温度和水 分是影响苔藓植物生存和分布的主要决定性因素, 尤其是空气湿度(娄红祥, 2012; Guo \& Zhao, 2018)。 党成强等(2018)研究表明, 南亚毛灰藓(Homomallium simlaense)在干旱和高温条件下, 各项生理指 标均与体内相对含水量呈正比。苔藓植物在干旱缺 水的情况下会停止生理代谢进入休眠状态, 当水分 充足时再进行生命活动。而且苔藓植物生物量与相 对生长速率随光照增强而增加, 随光照时间的延长, 其长度、 $\mathrm{pH}$ 值降低能力逐渐提高(St. Martin \& Mallik, 2017)。苔藓植物虽然能够忍受高温, 但是温度 高于 $25{ }^{\circ} \mathrm{C}$ 时却很少有净光合产物(Proctor, 2000; Hanson et al., 2014)。同时, 温度也是影响酶活性的 关键因素, 温度超过 $60{ }^{\circ} \mathrm{C}$ 时会严重影响 $\mathrm{CA}$ 的活性 (洪子茜, 2018)。然而, CA 参与苔藓植物岩溶作用的 最佳酶促反应温度还不得而知。同时, 温度也影响 有机酸的反应性、络合能力和岩石中矿物自身的溶 解程度(阊志为等, 2009; Benbouzid et al., 2012), 进 而影响岩石的溶蚀强度(陈传平等, 2008)。而且有机 酸与矿物反应生成有机酸盐的存在形态也随温度发 生变化(Peters et al., 2018)。

因此, 湿度、温度、光照及其综合因子通过对 苔藓植物生理代谢过程、基因型以及其他功能性状 的影响(Pharo \& Zartman, 2006; de la Rosa, 2016; 解 梦, 2018), 而直接或间接地影响苔藓植物岩溶作用 过程与效率。例如, 当温度在 $17.5-21.4{ }^{\circ} \mathrm{C}$, 湿度 $57.7 \%-72.6 \%$, 光照 2 700-5 $9001 x$ 条件下, 苔藓植 物对碳酸盐岩的塑造作用最强(申家琛等, 2018a)。 但是目前对温湿度及其综合因子对苔藓植物岩溶作 用的影响的关注度仍不够, 很多规律仍不清楚, 然 而这些规律的发现是揭示苔藓植物岩溶机理的关键, 也是指导岩溶生态问题治理的重要科学依据。

\section{$4.2 \mathrm{pH}$ 与其他营养元素}

Xie和Lou (2009)认为, 生物岩溶作用随 $\mathrm{pH}$ 值降 低而增强, 如果单纯从 $\mathrm{H}^{+}+\mathrm{CaCO}_{3} \rightleftharpoons \mathrm{CO}_{2}+\mathrm{H}_{2} \mathrm{O}+$ $\mathrm{Ca}^{2+}$ 角度分析pH对岩溶作用的影响, $\mathrm{pH}$ 值越低溶蚀 速率越快。然而岩石的最佳溶解 $\mathrm{pH}$ 由矿物自身属性 决定(Bray et al., 2015), 同时有机酸的溶蚀能力也 受pH影响(Pachana et al., 2012; Chen et al., 2016), 而且每类苔藓植物的最适生长 $\mathrm{pH}$ 也不尽相同。如泥 炭藓属(Sphagnum)植物适宜偏酸性环境, 细叶小羽 藓(Haplocladium microphyllum)配子体的最适生长 
$\mathrm{pH}$ 值为7 (陈文佳等, 2012)。作为苔藓植物岩溶作用 的重要参与者 $\mathrm{CA}$, 它的活性也受 $\mathrm{pH}$ 影响, 洪子茜 (2018) 研究表明, 当 $\mathrm{pH}$ 在 7-9 条件下 CA 的活性最 高。pH由外而内对苔藓植物岩溶作用的过程、速率、 对象、各级参与者都产生不同程度的促进或抑制作 用。目前我们对 $\mathrm{pH}$ 影响苔藓植物岩溶的规律以及调 节机制还不得而知, 今后还需综合全面地进行深入 研究。

由于苔藓植物属于贫营养型, 它所需要的营养 物质是维管束植物的 $1 / 10$, 但是对 $\mathrm{N} 、 \mathrm{P} 、 \mathrm{~K} 、 \mathrm{Ca} 、$ $\mathrm{Mg} 、 \mathrm{Fe}$ 、硫 $(\mathrm{S})$ 等元素的需求仍必不可少(娄红祥, 2012)。 $\mathrm{Ca}^{2+}$ 作为生物的第二信使, 具有调节生物抗 逆性等多种功能, 对苔藓植物的生长有显著影响 (Amao et al., 2010; Kolupaev et al., 2014)。当苔藓植 物缺 $\mathrm{Ca}$ 时会导致生长缓慢, 假根分化少, 原丝体不 发育, 叶状体形状改变等(娄红祥, 2012)。而且CA活 性也受 $\mathrm{Fe}^{2+}$ 、锌离子 $\left(\mathrm{Zn}^{2+}\right)$ 、硝酸根 $\left(\mathrm{NO}_{3}^{-}\right)$、氯离子 $\left(\mathrm{Cl}^{-}\right)$、澳离子 $\left(\mathrm{Br}^{-}\right)$、有机碳、交换性钙等的显著影 响(洪子茜, 2018)。例如, 黄芬等(2015)研究表明, Ca 对 CA的活性有很强的促进作用。生物与环境协同进 化致使某些苔藓出现钙依赖性, 这种对钙的依赖性 是否表现在基因水平, 从而影响岩溶地区苔藓植物 生物多样性、空间分布特征、功能性状等还有待进 一步研究。因此在研究苔藓植物岩溶作用与 $\mathrm{pH}$ 及营 养元素之间的关系时, 应综合考虑苔藓植物种类、 酶活性、逆境胁迫、水环境、基岩性质等因素。

\section{3 岩石性质}

岩石中所含矿物种类及其晶体结构、产状、透 水性等决定了岩石的溶解能力(Voinot et al., 2013; 黄奇波等, 2015), 例如, 一般情况下泥质含量高的 碳酸盐岩溶蚀速度快, 岩石中酸不溶物含量越低, 越易被溶解(王世杰等, 1999)。同时, 岩石中酸不溶 物含量对碳酸盐岩的成土速率具有重要影响, 酸不 溶物含量越高岩石风化后可残留物越多, 成土物质 越丰富(陈洪松等, 2018)。例如, 白云岩中酸不溶物 含量高, 发育的土层较石灰岩地区土层厚(陈洪松 等, 2013)。岩石中所含矿物的性质影响各元素的释 放速率, Andrew等(2015)在 $25{ }^{\circ} \mathrm{C} 、 \mathrm{pH}=2.6$ 的开放试 验系统与封闭试验系统内发现黑云母中的元素释放 按照 $\mathrm{Fe}>\mathrm{Mg}>\mathrm{Al}>\mathrm{Si}$ 的顺序进行, 且溶解速率符 合 $r_{i}=k_{\mathrm{H}, i} \times a_{\mathrm{H}^{+}}^{x i}, k_{\mathrm{H}}$ 为表面速率常数 $((2.15-30.6) \times$ $\left.10^{-10} \mathrm{~mole} \mathrm{~s}_{\text {biotite }} \cdot \mathrm{m}^{-2} \cdot \mathrm{s}^{-1}\right), a_{\mathrm{H}^{+}}$为 $\mathrm{H}^{+}$活性, $x_{i}$ 为矿物与
$\mathrm{H}^{+}$的反应级数 $(0.31-0.58)$; 分析认为, 岩石中各矿 物的溶解具有空间各向异性, 其边缘的反应效率强 于基面, 而且各个元素的释放具有一定的规律性。 苔藓植物种类的空间分布特征与岩性密切相关 (Gorozhankina \& Konstantinov, 2001), 而且岩性和 有机酸种类也会影响有机酸与岩石的反应速率 (Cama \& Ganor, 2006; Ghoorah et al., 2014; Mwangi et al., 2018), 从而导致它们岩溶作用的差异性。

\section{5 研究不足和展望}

苔藓植物岩溶作用是苔藓植物与岩石相互博峦 的动态过程。近年来苔藓植物岩溶作用取得了不少 研究进展, 主要集中在苔藓持水特性与岩溶作用的 关系, 生物酶对溶蚀作用的影响, 苔藓植物及其生 物结皮的成土作用、钙华沉积以及苔藓植物溶蚀微 地貌及其与生境间的相互关系探讨方面。但也存在 不足之处，以下 4 个方面更为突出。

\section{1 苔藓植物岩溶作用内在机制与过程}

目前, 苔藓植物岩溶作用以定性描述为主, 溶 蚀作用发生的机理、条件, 哪些物质参与溶蚀? 这 些物质与岩石的反应过程、化学键的断裂与重组方 式、能量释放、产物的定性与定量分析, 以及它们 的溶蚀速率与成土速率如何? 不同生活型或不同物 种之间是否存在岩溶作用的差异性? 这些基础而又 关键的问题仍不清楚。因此, 今后需要加强苔藓植 物岩溶作用效率、驱动机制及其动态变化等内在机 制与过程的研究。

\section{2 苔藓植物岩溶作用与生境间互作机制}

苔藓植物岩溶作用与环境之间的三向循环互作 机制如图3所示，苔藓、环境因子、岩石三者之间相 互联系、相互作用。申家琛等(2018b)阐释了苔藓植 物溶蚀微地貌发育及其分布特征与生境(温湿度、光 照等)的关系, 但是对苔藓植物岩溶作用对生境的 改造, 以及生态因子对苔藓植物岩溶作用的影响还 未揭示。因此, 今后需要在这方面开展以下研究工 作: (1)温度、湿度、光照、 $\mathrm{pH}$ 、地质背景等因子对 苔藓植物生物多样性、生存繁殖, 生理调节机制、 功能性状等的影响; (2)环境因子对苔藓植物溶蚀过 程、效率、物质能量转化过程等的影响; (3)苔藓植 物沉积过程对岩面微环境的温湿度、地貌塑造、土 壤养分、水动力特征等的影响; (4)苔藓植物与其内 生菌和藻类的共生关系以及与其他植物之间的化感 


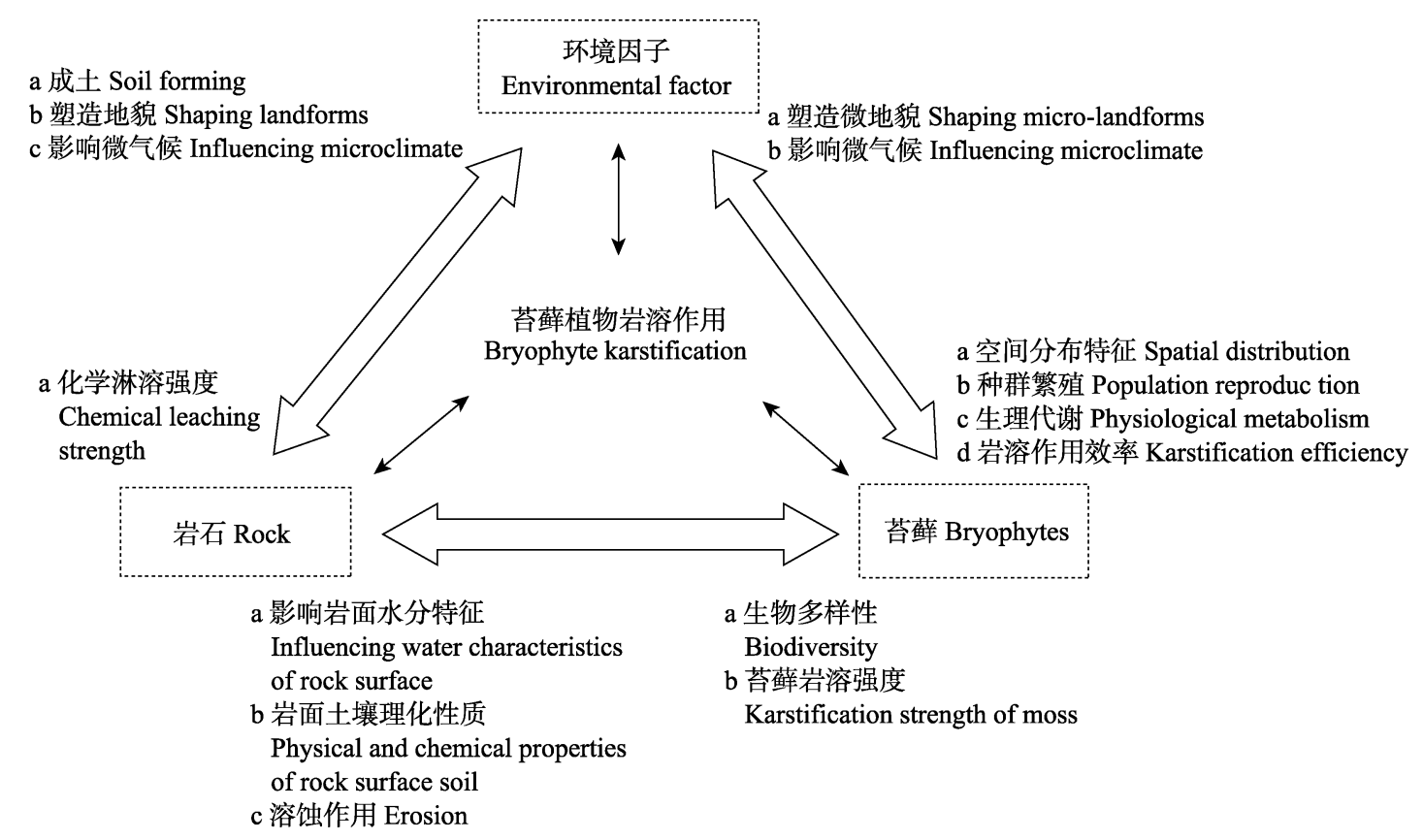

图3 苔藓植物岩溶作用与环境、岩石间的三向循环互作机制图。苔藓、环境因子、岩石在岩溶作用过程中三者既相互促进 又相互制约。环境因子与岩石通过控制苔藓植物的群落特征、形态、生理过程、基因等影响岩溶过程。反之基岩的岩性、组 成、产状，以及苔藓植物对岩面生境(温度、湿度、光照、土壤肥力、微生物)的改善与岩溶速率密切相关。

Fig. 3 The three-way circulation interaction mechanism among bryophyte karstification, environment and rock. Bryophyte, environmental factors and rock in the karstification process promote and restraint each other. Environmental factors and rock affect karstification process and efficiency by controlling the community characteristics, morphology, physiological processes, genes, etc. of bryophyte. The lithology, composition, occurrence of rock, and the improvement of bryophyte on rock surface habitat (temperature, humidity, light, soil fertility, microbes) are closely related to the rate of karstification.

作用对岩溶作用的影响。

\section{3 苔藓植物的生理代谢过程及其生物酶等与岩 溶作用的相互关系}

苔藓植物体内的CA对岩溶作用有促进作用, 但是CA活性存在显著的种间差异，如何通过植物 生理或者基因学调控 $\mathrm{CA}$ 代谢过程，提高酶活性? 苔藓植物呼吸作用产生的 $\mathrm{H}_{2} \mathrm{CO}_{3}$ 与代谢分泌物的溶 蚀作用差异性及相互关系? 分泌物的代谢调控机 制? 这些科学问题的阐释对揭示苔藓植物岩溶作用 机理、调控岩溶过程等具有重要的理论指导意义。

\section{4 岩溶地区苔藓植物环境适应性特征及其生物 多样性维持机制}

土层浅薄、贫㾑、钻丰富是岩溶生态系统的典 型特征。苔藓植物与岩溶生境在长期协同进化过程 中形成了与环境相适应的功能性状特征。目前, 对 岩溶地区苔藓植物环境适应性特征与生态系统过程 和功能关系的认识还十分有限, 无法揭示岩溶生态 系统生物多样性维持机制。因此, 需要开展苔藓植 物形态结构特征、生理调节机制(光合、呼吸、抗逆 性、物质代谢等)、养分需求及利用方式、繁殖方式、
群落组成结构特征、物种多样性、遗传多样性等与 岩溶生态系统结构、功能、生物多样性之间的互作 机制。同时，植物功能性状与生态系统间的耦合效 应会成为今后植物生态学研究的热点问题。

此外, 建议在苔藓植物岩溶作用研究时建立野 外长期监测基地，监测苔藓植物对全球变化(增温、 降水格局、氮沉降等)响应及其对岩溶作用的影响。 同时, 通过同位素示踪法研究苔藓植物岩溶作用过 程中碳循环路径, 建立大气-水环境-生物-土壤-基 岩“五位一体”的碳循环研究模式, 进而为研究岩溶 生态系统碳循环、 $\mathrm{CO}_{2}$ 收支与排放量、碳汇问题、 全球变暖等提供新思路、新方法。

致谢 感谢贵州省林业厅攻关项目([2016]12号)和 贵州科学院青年重点基金([2020]03号)资助。

\section{参考文献}

Amao Y, Takai K, Ohashi A (2010). Effect of manganese and calcium ions on the photoinduced water oxidation with photosynthesis organ grana from green plant. Applied $\mathrm{Ca}$ talysis B: Environmental, 97, 36-40.

Benbouzid H, Le Floch S, Stephan L, Olier R, Privat M (2012). 
Combined effects of salinity and temperature on the solubility of organic compounds. The Journal of Chemical Thermodynamics, 48, 54-64.

Bray AW, Oelkers EH, Bonneville S, Wolff-Boenisch D, Potts NJ, Fones G, Benning LG (2015). The effect of $\mathrm{pH}$, grain size, and organic ligands on biotite weathering rates. Geochimica et Cosmochimica Acta, 164, 127-145.

Cai HL (2017). Surface Dissolved Forms of Carbonate Rocks and Its Influencing Factors. Master degree dissertation, Yunnan Normal University, Kunming. [蔡胡霖 (2017). 碳酸盐岩表面溶蚀形态及其影响因素. 硕士学位论文, 云南师范大学, 昆明.]

Cama J, Ganor J (2006). The effects of organic acids on the dissolution of silicate minerals: A case study of oxalate catalysis of kaolinite dissolution. Geochimica et Cosmochimica Acta, 70, 2191-2209.

Cao JH, Wang FX (1996). Observations on fractal characters between karst macrophology and biokarst micromorphology in the Guilin area. Journal of Nanjing University (Natural Science), 32, 146-147. [曹建华, 王福星 (1996). 桂林地区生物岩溶微观形态与岩溶地貌宏观形态间的 分形特征. 南京大学学报(自然科学版), 32, 146-147.]

Cao JH, Yuan DX (1999). Relationship between water-holding of carbonate rock and saxicolous algae, lichen and moss and its ecological significance. Geochimica, 28, 248-256. [曹建华, 袁道先 (1999). 石生藻类、地衣、苔藓与碳酸 盐岩持水性及生态意义. 地球化学, 28, 248-256.]

Cao JH, Yuan DX, Zhang C, Jiang ZC (2004). Karst ecosystem constrained by geological conditions in southwest China. Earth and Environment, 1, 1-8. [曹建华, 袁道先, 章程, 蒋忠诚 (2004). 受地质条件制约的中国西南岩溶生态 系统. 地球与环境, 1, 1-8.]

Cao QZ, Deng M, Huang B, Chen B (2018). Influence of acid-insoluble residue on dedolomite reaction in dolomitic rocks. Journal of Nanjing Tech University (Natural Science Edition), 40, 89-94. [曹沁智, 邓敏, 黄蓓, 陈碧 (2018). 白云质岩石中酸不溶物对碱白云石反应的影响. 南京工业大学学报(自然科学版), 40, 89-94.]

Chen CP, Gu X, Zhou SM, Liu JP (2008). Experimental research on dissolution dynamics of main minerals in several aqueous organic acid solutions. Acta Geologica Sinica，7，1007-1012. [陈传平，固旭，周苏闽，刘建平 (2008). 不同有机酸对矿物溶解的动力学实验研究. 地 质学报, 7, 1007-1012.]

Chen HS, Fu ZY, Zhang W, Nie YP (2018). Soil water processes and vegetation restoration in karst region of southwest China. Chinese Journal of Nature, 40, 41-46. [陈洪 松, 付智勇, 张伟, 聂云鹏 (2018). 西南喀斯特地区水 土过程与植被恢复重建. 自然杂志, 40, 41-46.]

Chen HS, Nie YP, Wang KL (2013). Spatio-temporal heterogeneity of water and plant adaptation mechanisms in karst regions: A review. Acta Ecologica Sinica, 33, 317-326. [陈洪松, 聂云鹏, 王克林 (2013). 岩溶山区水分时空 异质性及植物适应机理研究进展. 生态学报, 33 , 317-326.]

Chen WJ, Zhang N, Hang LL, Wang Y, Ji MC (2012). Effects of different medium and $\mathrm{pH}$ value on the growth of gametophyte in Haplocladium microphyllum. Practical Forestry Technology, (4), 20-23. [陈文佳, 张楠, 杭璐璐, 王媛, 季梦成 (2012). 不同培养基及 $\mathrm{pH}$ 值对细叶小羽 藓配子体生长的影响. 林业实用技术, (4), 20-23.]

Chen ZY, Liu F, Bu TD, Liu YS, Zhu J (2016). Effects of organic acids on dissolution of $\mathrm{Fe}$ and $\mathrm{Mn}$ from weathering coal gangue. Acta Geochimica, 3, 316-328.

Costa DP, Amado-Filho GM, Pereira RC, Paradas WC, Miyataka H, Okamoto Y, Asakawa Y (2018). Diversity of secondary metabolites in the liverwort Syzygiella rubricaulis (Nees) Stephani (Jamesoniellaceae, Marchantiophyta) from neotropical high mountains. Chemistry \& Biodiversity, 15, e1800239. DOI: 10.1002/cbdv.201800239.

Dang CQ, Li ZF, Chen M, Gao T, Huang HM, Liu JC, Tao JP (2018). Physiological and biochemical characteristics of epilithic moss Homomallium simlaense (Mitt.) Broth. Mitt under high temperature and drought stress. Plant Science Journal, 36, 393-401. [党成强, 李宗峰, 陈沝, 高婷, 黄 慧敏, 刘锦春, 陶建平 (2018). 石生南亚毛灰藓在不同 温度和干旱条件下的生理生化特性. 植物科学学报, 36 , 393-401.]

de la Rosa JPM (2016). The Burren: A glacial, karstic and biokarstic expression of a limestone plateau in western Ireland. Earth Surface Processes and Landforms, 41, 1614-1628.

Fu L, Zhang ZH (2010). Preliminary study on biokarst erosion of bryophytes in Guiyang city. Journal of Guizhou Normal University (Nature Science), 28(4), 140-143. [付兰, 张朝 晖 (2010). 贵阳市苔藓植物的生物岩溶溶蚀初探. 贵州 师范大学学报(自然科学版), 28(4), 140-143.]

Ghoorah M, Dlugogorski BZ, Balucan RD, Kennedy EM (2014). Selection of acid for weak acid processing of wollastonite for mineralisation of $\mathrm{CO}_{2}$. Fuel, 122, 277-286.

Gorozhankina SM, Konstantinov VD (2001). Comparative ecocenotic characteristics of mosses in the taiga zone of West Siberia. Russian Journal of Ecology, 32, 386-392.

Guo Y, Wang ZH, Zhang ZH (2018). Study on community characteristics of bryophytes in dolomite cave twilight zones: An example of the Shuidong Cave in Suiyang county, Guizhou Province. Carsologica Sinica, 37, 388-399. [郭云, 王智慧, 张朝晖 (2018). 白云岩洞穴 洞口弱光带的苔藓群落特征——绥阳水洞为例. 中 国岩溶, 37, 388-399.]

Guo YW, Zhao YG (2018). Effects of storage temperature on the physiological characteristics and vegetative propagation 
of desiccation-tolerant mosses. Biogeoscience, 15, 797-808.

Hanson DT, Renzaglia K, Villarreal JC (2014). Diffusion limitation and $\mathrm{CO}_{2}$ concentrating mechanisms in bryophytes. In: Hanson DT, Rice SK eds. Photosynthesis in Bryophytes and Early Land Plants. Springer, Dordrecht, Netherlands. 95-111.

Harper KT, Belnap J (2001). The influence of biological soil crusts on mineral uptake by associated vascular plants. Journal of Arid Environments, 47, 347-357.

Haward SJ, Smits MM, Ragnarsdóttir KV, Leake JR, Banwart SA, McMaster TJ (2011). In situ atomic force microscopy measurements of biotite basal plane reactivity in the presence of oxalic acid. Geochimica et Cosmochimica Acta, $75,6870-6881$.

Heino J, Virtanen R (2006). Relationships between distribution and abundance vary with spatial scale and ecological group in stream bryophytes. Freshwater Biology, 51, 1879-1889.

Hong ZX (2018). Study on the characteristics of microbial carbonic anhydrase. Biological Chemical Engineering, (4), 63-66. [洪子茜 (2018). 微生物碳酸酐酶特性研究. 生 物化工, (4), 63-66.]

Huang F, Huang YM, Gao X, Cao JH (2015). Effects of karst environmental factors on activity of soil microorganic extracellular carbonic anhydrase of karst area in Maocun village, Guilin. Journal of Southern Agriculture, 46, 1792-1797. [黄芬, 黄艳梅, 高喜, 曹建华 (2015). 岩溶 环境因子对桂林毛村岩溶区土壤微生物胞外碳酸酐酶 活性的影响. 南方农业学报, 46, 1792-1797.]

Huang QB, Qin XQ, Liu PY, Lan FN, Zhang LK (2015). Dissolution rate and it's significance of different lithological tablets. Earth and Environment, 43, 379-385. [黄奇波, 覃 小群, 刘朋雨, 蓝芙宁, 张连凯 (2015). 不同岩性试片 溶蚀速率差异及意义. 地球与环境, 43, 379-385.]

Jackson TA (2015). Weathering, secondary mineral genesis, and soil formation caused by lichens and mosses growing on granitic gneiss in a boreal forest environment. Geoderma, 251, 78-91.

Jia SH, Li JF, Wang ZH, Zhang ZH (2014). Ecological function of bryophyte on karst rocky desertification slopes along mountainous roads. Chinese Journal of Ecology, 33, 1928-1934. [贾少华, 李军峰, 王智慧, 张朝晖 (2014). 岩溶山区公路石漠化边坡苔藓生态功能. 生态学杂志, 33, 1928-1934.]

Kleinteich J, Golubic S, Pessi IS, Velázquez D, Storme J-Y, Darchambeau F, Borges AV, Compère P, Radtke G, Lee S-J, Javaux EJ, Wilmotte A (2017). Cyanobacterial contribution to travertine deposition in the Hoyoux River system, Belgium. Microbial Ecology, 74, 33-53.

Kolupaev YE, Vayner AA, Yastreb TO, Oboznyi AI, Khripach www.plant-ecology.com
VA (2014). The role of reactive oxygen species and calcium ions in the implementation of the stress protective effect of brassinosteroids on plant cells. Applied Biochemistry and Microbiology, 50, 658-663.

Krol E, Dziubinska H, Trebacz K (2003). Low-temperature induced transmembrane potential changes in the liverwort Conocephalum conicum. Plant \& Cell Physiology, 44, 527-533.

Lammers K, Smith MM, Carroll SA (2017). Muscovite dissolution kinetics as a function of $\mathrm{pH}$ at elevated temperature. Chemical Geology, 466, 149-158.

Li JF, Wang ZH, Zhang ZH (2013). Bryophyte diversity and the effect of soil formation along with water conservation in karst rocky desertification region. Research of Environmental Sciences, 26, 759-764. [李军峰, 王智慧, 张朝 晖 (2013). 喀斯特石漠化山区苔藓多样性及水土保持 研究. 环境科学研究, 26, 759-764.]

Li T, Wang HY, Zhou ZJ, Chen XQ, Zhou JM (2015). A nano-scale study of the mechanisms of non-exchangeable potassium release from micas. Applied Clay Science, 118, 131-137.

Li W, Yu LJ, Yu JF, Jia LP, Wu Y (2005). Effects of karst environmental factors on expression and activity of bacterial extracellular carbonic anhydrase. Microbiology China, 5, 35-39. [李为, 余龙江, 余俊峰, 贾丽萍, 吴云 (2005). 岩溶环境因子对细菌胞外碳酸酐酶表达及活性的影响. 微生物学通报, 5, 35-39.]

Li W, Yu LT, Wu Y, Jia LP, Yuan DX (2006). Enhancement of $\mathrm{Ca}^{2+}$ release from limestone by microbical extracelluar carbonic anhydrase. Bioresource Technology, 98, 950-953.

Li ZK, Wu QM, Wang ZH, Zhang ZH (2018). Diversity and ecological characteristics of bryophytes from peak cluster under the background of karst urban. Bulletin of Botanical Research, 38, 433-443. [李泽科, 吴启美, 王智慧, 张朝 晖 (2018). 岩溶城市背景下贵阳市峰丛苔藓植物多样 性分布及其生态特征. 植物研究, 38, 433-443.]

Lian B, Chen Y, Zhu LJ, Yang RD (2008). Progress in the study of the weathering of carbonate rock by microbes. Earth Science Frontiers, 15, 90-99. [连宾, 陈华, 朱立军, 杨瑞东 (2008). 微生物对碳酸盐岩的风化作用. 地学前 缘, 15, 90-99.]

Lian B, Yuan DX, Liu ZH (2011). Effect of microbes on karstification in karst ecosystems. Chinese Science Bulletin, 56, 2158-2161. [连宾, 袁道先, 刘再华 (2011). 岩溶生态 系统中微生物对岩溶作用影响的认识. 科学通报, 56, 2158-2161.]

Liang FY, Xu B (2014). Discrimination of tower, cockpit, and non-karst landforms in Guilin, Southern China, based on morphometric characteristics. Geomorphology, 204, 42-48.

Liu MX, Xu XL, Wang DB, Alexander YS, Wang KL (2016). Karst catchments exhibited higher degradation stress from 
climate change than the non-karst catchments in southwest China: An ecohydrological perspective. Journal of $\mathrm{Hy}$ drology, 535, 173-180.

Liu SL, Zhu SL, Li J, Yang Y, Li JG (2017). A study on the ability of different organic acids to dissolve tricalcium phosphate. Acta Agriculturae Universitatis Jiangxiensis, 39, 1010-1016. [刘胜亮, 朱舒亮, 李静, 杨越, 李建贵 (2017). 不同有机酸对磷酸三钙溶解能力的研究. 江西 农业大学学报, 39, 1010-1016.]

Liu TL, Cong CL, Hu D, Wang SJ, Zhang XQ (2017). Carbonic anhydrase activity of six epilithic mosses and their underlying soil in the Puding karst area, Guizhou Province. Carsologica Sinica, 36, 187-192. [刘天雷, 从春蕾, 胡 丹, 王世杰, 张显强 (2017). 贵州普定6种喀斯特石生 植物及其土壤的碳酸䣶酶活性. 中国岩溶, 36, 187-192.]

Liu Y, Pi CY, Tian S (2015). Relationships between characteristics of ground bryophyte communities and environmental factors in urban area of Chongqing, China. Chinese Journal of Applied Ecology, 26, 3145-3152. [刘艳，皮春燕， 田尚 (2015). 重庆主城区地面苔藓植物群落特征及其 与环境的关系. 应用生态学报, 26, 3145-3152.]

Lou HX (2012). Bryophyte Chemistry and Biology. Science Press, Beijing. [娄红祥 (2012). 苔蘚化学与生物学. 科 学出版社, 北京.]

Lou YX (2013). Study on Response Mechanism and Biological Indicators of Bryophytes to Heavy Metal Pollution. $\mathrm{PhD}$ dissertation, Shanghai Normal University, Shanghai. [娄玉 霞 (2013). 苔藓植物对重金属污染的响应机理和生物 指示的研究. 博士学位论文, 上海师范大学, 上海.]

Miliša M, Habdija I, Primc-Habdija B, Radanović I, Kepčija RM (2006). The role of flow velocity in the vertical distribution of particulate organic matter on moss-covered travertine barriers of the Plitvice Lakes (Croatia). Hydrobiologia, 553, 231-243.

Mod HK, Heikkinen RK, le Roux PC, Väre H, Luoto M (2016). Contrasting effects of biotic interactions on richness and distribution of vascular plants, bryophytes and lichens in an arctic-alpine landscape. Polar Biology, 39, 649-657.

Mwangi P, Brady PV, Radonjic M, Thyne G (2018). The effect of organic acids on wettability of sandstone and carbonate rocks. Journal of Petroleum Science \& Engineering, 165, 428-435.

Pachana K, Zuddas P, Censi P (2012). Influence of pH and temperature on the early stage of mica alteration. Applied Geochemistry, 27, 1738-1744.

Pang JP, Wang ZH, Zhang ZH (2018). Characteristics of bryophyte communities and their successional patterns in different habitats from karst dolomite rocky desertification areas. Ecological Science, 37, 59-66. [庞嘉鹏, 王智慧, 张朝晖 (2018). 喀斯特白云岩石漠化区域不同生境条
件下苔藓植物群落特征及演替模式研究. 生态科学, 37 , 59-66.]

Peters K, Gorzolka K, Bruelheide H, Neumann S (2018). Seasonal variation of secondary metabolites in nine different bryophytes. Ecology and Evolution, 8, 9105-9117.

Pharo EJ, Zartman CE (2006). Bryophytes in a changing landscape: The hierarchical effects of habitat fragmentation on ecological and evolutionary processes. Biological Conservation, 135, 315-325.

Proctor MCF (2000). The bryophyte paradox: Tolerance of desiccation, evasion of drought. Plant Ecology, 151, 41-49.

Proctor MCF, Pence VC (2002). Vegetative tissues: Bryophytes vascular "resurrection plants" and vegetative propagules. In: Pritchard H, Back M eds. Desiccation and Plant Survival. CABI Publishing, Wallingford, UK. 207-237.

Schneider J (1976). Biological and inorganic factors in the destruction of limestone coasts. Contributions to Sedimentology, 6, 112-116.

Shen JC, Zhang ZH, Wang HH, Huang H, Wang ZH (2017). Water retention capacity of autumn mosses in south stone forest of Guiyang karst park. Journal of Ecology and Rural Environment, 33, 907-912. [申家琛, 张朝晖, 王慧慧, 黄欢, 王智慧 (2017). 贵阳喀斯特公园南石林秋季藓类 植物的持水特性. 生态与农村环境学报, 33, 907-912.]

Shen JC, Zhang ZH, Wang HH, Huang H, Wang ZH (2018a). Corrosion effects and environmental correlation of bryophytes on limestone in Guiyang karst park. Carsologica Sinica, 37, 175-184. [申家琛, 张朝晖, 王慧慧, 黄欢, 王智慧 (2018a). 苔藓植物对石灰岩的溶蚀作用及环境 相关性研究. 中国岩溶, 37, 175-184.]

Shen JC, Zhang ZH, Wang ZH (2018b). The effects of rocky desertification degree on bryophyte diversity and soil chemical properties of crusts. Acta Ecologica Sinica, 38, 6043-6054. [申家琛, 张朝晖, 王智慧 (2018b). 石漠化 程度对苔藓植物多样性及其结皮土壤化学性质的影响. 生态学报, 38, 6043-6054.]

Shen TM (2018). Contribution and Mechanism of Microbes and Carbonic Anhydrase to Carbon Storage Soil Ecosystems. PhD dissertation, Huazhong University of Science and Technology, Wuhan. [申泰铭 (2018). 微生物及碳酸 酕酶对岩溶土壤生态系统碳储存的贡献及其机制. 博 士学位论文, 华中科技大学, 武汉.]

Shen TM, Xin BG, Li W, Yu LJ (2014). Characteristics of carbonate rock corrosion by different kinds of microbea and their carbonic anhydras in the $\mathrm{CO}_{2}-\mathrm{H}_{2} \mathrm{O}$-carbonate system. Bulletin of Mineralogy, Petrology and Geochemistry, 33, 797-800. [申泰铭, 邢必果, 李为, 余龙江 (2014). 不同 种类微生物及其碳酸酐酶对 $\mathrm{CO}_{2}-\mathrm{H}_{2} \mathrm{O}$-碳酸盐系统中碳 酸盐岩的溶蚀作用. 矿物岩石地球化学通报, 33, 797-800.] 
Smith EC, Griffiths H (2000). The role of carbonic anhydrase in photosynthesis and the activity of the carbonconcentrating-mechanism in bryophytes of the class anthocerotae. New Phytologist, 145, 29-37.

St. Martin P, Mallik AU (2017). The status of non-vascular plants in trait-based ecosystem function studies. Perspectives in Plant Ecology, Evolution and Systematics, 27, 1-8.

Street LE, Subke J-A, Sommerkorn M, Sloan V, Ducrotoy H, Phoenix GK, Williams M (2013). The role of mosses in carbon uptake and partitioning in arctic vegetation. New Phytologist, 199, 163-175.

Voinot A, Lemarchand D, Collignon C, Granet M, Chabaux F, Turpault M-P (2013). Experimental dissolution vs. transformation of micas under acidic soil conditions: Clues from boron isotopes. Geochimica et Cosmochimica Acta, 117, 144-160.

Wang FX, Cao JH, Huang JF (1998). Biokarst in the cave twilight zones. Carsologica Sinica, 17, 42-48. [王福星, 曹建 华, 黄俊发 (1998). 洞穴弱光带的生物岩溶. 中国岩溶, 17, 42-48.]

Wang FX, Cao JH, Huang JF, Jiang LD, Huang JF, Wang J (1993). Biokarst. Geological Publishing House, Beijing. [王福星, 曹建华, 黄俊发, 江利登, 黄基富, 王晶 (1993). 生物岩溶. 地质出版社, 北京.]

Wang GM, Xiong ZH, Zhang J, Zhang B (2017). Dissolution experiment and transformation condition analysis of $\mathrm{Pa}$ leogene aragonite in the Jiyang Depression, China. Australian Journal of Earth Sciences, 64, 343-352.

Wang HJ, Yan H, Liu ZH (2014). Contrasts in variations of the carbon and oxygen isotopic composition of travertines formed in pools and a ramp stream at Huanglong Ravine, China: Implications for paleoclimatic interpretations. Geochimica et Cosmochimica Acta, 125, 34-48.

Wang SJ, Ji HB, Ouyang ZY, Zhou DQ, Zheng LP, Li TY (1999). Preliminary study on weathering and soil formation of carbonate rocks. Science in China (Series D), 29, 441-449. [王世杰, 季宏兵, 欧阳自远, 周德全, 郑乐平, 黎廷宇 (1999). 碳酸盐岩风化成土作用的初步研究. 中 国科学(D辑: 地球科学), 29, 441-449.]

Won-Pyo P, Bon-Jun K, Chang AC, Ferko TE, Parker JR, Ward TH, Lara SV, Nguyen CM (2016). Dissolution of metals from biosolid-treated soils by organic acid mixtures. Applied and Environmental Soil Science, 2016, $1-15$.

Wu YH, Huang GH, Gao Q, Cao T (2001). Research advance in response and adaptation of bryophytes to environmental change. Chinese Journal of Applied Ecology, 12, 943-946. [吴玉环, 黄国宏, 高谦, 曹同 (2001). 苔藓植物对环境 变化的响应及适应性研究进展. 应用生态学报, 12 , 943-946.]

Wu YW, Zhang JC (2015). Microbial carbonic anhydrase ac- tion and application on carbon cycling in karst dynamic system: A review. Journal of Biology, 32, 78-83. [吴雁雯, 张金池 (2015). 微生物碳酸酐酶在岩溶系统碳循环中 的作用与应用研究进展. 生物学杂志, 32, 78-83.]

Xie CF, Lou HX (2009). Secondary metabolites in bryophytes: An ecological aspect. Chemistry \& Biodiversity, 6, 303-312.

Xie M (2018). Determination of DNA C-values and Analysis of Variation Characteristics of Bryophytes. Master degree dissertation, Shanghai Normal University, Shanghai. [解梦 (2018). 苔藓植物DNA C-值测定及其变异特点分析. 硕 士学位论文, 上海师范大学, 上海.]

Xue JH (2013). Forest Ecology. China Forestry Publishing House, Beijing. [薛建辉 (2013). 森林生态学. 中国林业 出版社, 北京.]

Yan H, Liu ZH, Sun HL (2017). Effect of in-stream physicochemical processes on the seasonal variations in $\delta^{13} \mathrm{C}$ and $\delta^{18} \mathrm{O}$ values in laminated travertine deposits in a mountain stream channel. Geochimica et Cosmochimica Acta, 202, 179-189.

Yan ZW, Liu HL, Zhang ZW (2009). Influences of temperature and $\mathrm{P}_{\mathrm{CO}_{2}}$ on the solubility of calcite and dolomite. Carsologica Sinica, 28，7-10. [闵志为, 刘辉利, 张志卫 (2009). 温度及 $\mathrm{CO}_{2}$ 对方解石、白云石溶解度影响特征分 析. 中国岩溶, 28, 7-10.]

Zhang DG, Li J, Liu H (2015). Effect of exogenous low molecular weight organic acids on calcium migration in soil. Journal of Soil and Water Conservation, 29, 152-155. [张 大庚, 栗杰, 刘慧 (2015). 外源低分子量有机酸对土壤 中钻素迁移特征的影响. 水土保持学报, 29, 152-155.]

Zhang J (1993). Biological role in the formation of karst barrier lakes in Mianshan limestone area, northwest Sichuan. Journal of Lake Sciences, 5, 33-39. [张捷 (1993). 川西 北眠山灰岩区岩溶堰塞湖形成中的生物作用. 湖泊科 学, 5, 33-39.]

Zhang KY (2017). The Dissolution of Limestone by Some of the Mosses and the Microorganism in Karst Area in Guizhou Province. Master degree dissertation, Southwestern University, Chongqing. [张楷燕 (2017). 贵州喀斯特几种石 生苔藓和土壤微生物对石灰岩的溶蚀作用. 硕士学位 论文, 西南大学, 重庆.]

Zhang ML, Deng ZQ (1994). The soil and soil-forming processes in karst area of South China. Journal Guizhou Institute of Technology, 23, 67-75. [张美良, 邓自强 (1994). 我国南方岩溶地区的土壤及其形成. 贵州工学院学报, 23, 67-75.]

Zhang XQ, Liu TL, Cong CL (2018a). Study on soil conservation and pedogenic function of five bryophytes in the karst areas of Guizhou Province. Carsologica Sinica, 37, 708-713. [张显强, 刘天雷, 从春蕾 (2018a). 贵州5种喀 斯特石生藓类成土及保土生态功能研究. 中国岩溶, 37 ,

www.plant-ecology.com 
708-713.]

Zhang XQ, Long HY, Liu TL, Cong CL (2018b). Comparison of water absorption characteristic and water retention capacity of five epilithic mosses in the karst areas of Guizhou Province. Carsologica Sinica, 37, 835-841. [张显强, 龙华英, 刘天雷, 从春蕾 (2018b). 贵州喀斯特地区5 种 石生藓类的持水性能及吸水特征比较. 中国岩溶, 37 , 835-841.]

Zhang XQ, ZhaoYZ, Wang SJ (2017). Responses of antioxidant defense system of epilithic mosses to drought stress in karst rock desertified areas. Acta Geochimica, 36, 205-212.

Zhang ZH, Chen JK (2007). Floristic characteristics of aquatic bryophytes and their biokarst deposition types at waterfalls in central Guizhou, China. Carsologica Sinica, 26, 171-177. [张朝晖, 陈家宽 (2007). 黔中瀑布水生苔藓 植物区系及其生物喀斯特沉积生态类型研究. 中国岩 溶, 26, 171-177.]

Zhang ZH, Peng T, Li XN, Zhao CH (2004). Bryophytes in karst cave entrance zone of Kunming, China. Carsologica Sinica, 23, 230-233. [张朝晖, 彭涛, 李晓娜, 赵传海
(2004). 中国昆明地区岩溶洞穴洞口带苔藓植物研究. 中国岩溶, 23, 230-233.]

Zhao ZM, Luan Y, Quan SC, Wu JL, Liang Y, Zeng Z (2018). Effect of functional groups for the organic acid on the crystal transition of phosphogypsum crystal. Journal of Building Materials, 21, 247-252. [赵志曼, 奕扬, 全思臣, 吴佳丽, 梁祎, 曾众 (2018). 含辅助官能团类有机酸对 磷石亳晶体的影响. 建筑材料学报, 21, 247-252.]

Zheng YP, Zhao JC, Zhang BC, Li L, Zhang YM (2009). Research progress of algae and bryophytes in desert biological crusts. Journal of Integrative Plant Biology, 44, 371-378. [郑云普, 赵建成, 张丙昌, 李琳, 张元明 (2009). 荒漠生物结皮中藻类和苔蘚植物研究进展. 植 物学报, 44, 371-378.]

Zhu XM (1995). The process of the original soil formation. Research of Water and Soil Conservation, 4, 83-89. [朱显 谟 (1995). 论原始土壤的成土过程. 水土保持研究, 4, 83-89.]

责任编委: 郭 柯 责任编辑: 李 敏 实习编辑: 赵 航 